\title{
1 Characterization and Stability of Anthocyanins in Purple-fleshed Sweet Potato P40
}

2 Jianteng $\mathrm{Xu}^{\mathrm{a}}$, Xiaoyu Su${ }^{\mathrm{a}}$, Soyoung Lim ${ }^{\mathrm{a}}$, Jason Griffin ${ }^{\mathrm{b}}$, Edward Carey ${ }^{\mathrm{c}}$, Benjamin Katz ${ }^{\mathrm{d}}$, John

3 Tomich $^{\mathrm{d}}$, J. Scott Smith ${ }^{\mathrm{e}}$, Weiqun Wanga,*

$4{ }^{a}$ Department of Human Nutrition, ${ }^{b}$ Department of Horticulture, ${ }^{d}$ Department of Biochemistry,

$5 \quad$ Institute of Food Science, Kansas State University, Manhattan, KS 66506;

$6 \quad$ International Potato Center, Kumasi, Ghana

7

8 * Corresponding author. Telephone: 785-532-0153. Fax: 785-532-3132. E-mail:

9 wwang@ksu.edu

10 


\section{Abstract}

Purple-fleshed sweet potato P40 has been shown to prevent colorectal cancer in a murine

13 model. This study is to identify anthocyanins by using HPLC/MS-MS and assess the stability

14 during various cooking conditions. P40 possesses a high content of anthocyanins up to $14 \mathrm{mg} / \mathrm{g}$

15 dry matter. Total 12 acylated anthocyanins are identified. Top three anthocyanins, e.g., cyanidin

16 3-caffeoyl-p-hydroxybenzoyl sophoroside-5-glucoside, peonidin 3-caffeoyl sophoroside-5-

17 glucoside, and cyanidin 3-(6" -caffeoyl-6"'-feruloylsophoroside)-5-glucoside, account for half

18 of the anthocyanin contents. Over $80 \%$ of anthocyanins measured by acid hydrolysis were

19 cyanidin derivatives, indicating P40 is unique when compared with other purple-fleshed sweet

20 potatoes that usually contain more peonidin than cyanidin. Steaming, pressure cooking,

21 microwaving, and frying but not baking significantly reduced 8-16\% of total anthocyanin

22 contents. Mono-acylated anthocyanins showed a higher resistance against heat than di- and non-

23 acylated. Among of which, cyanidin 3-p-hydroxybenzoylsophoroside-5-glucoside exhibited the

24 best thermal stability. The stable acylated and cyanidin-predominated anthocyanins in P40 may

25 provide extra benefits for cancer prevention.

26 Keywords: Anthocyanins / purple-fleshed sweet potato / cancer prevention / stability / cooking

27 conditions 


\section{Introduction}

Sweet potato (Ipomoea batatas) is known as an excellent source of $\beta$-carotene (precursor of vitamin A), vitamin Bs, dietary fiber, minerals, and polysaccharides. In year 2003, the global

31 production of sweet potato was estimated about 122 million metric tons, which was heavily

32 consumed in rural part of China and western African countries (Wu et al., 2008). Purple-fleshed

33 sweet potato (PSP) contains a significantly greater amount of anthocyanin than ordinary orange-

34 fleshed sweet potato. Through several years, a number of PSP varieties with different

35 anthocyanin contents and profiles were bred and grown for their potential health benefits.

36 Differing from anthocyanins found in berries, PSP anthocyanins primarily exist as

37 acylated forms (Giusti \& Wrolstad, 2003; Gould, Davies \& Winefield, 2008). Acylation with

38 various phenolic acids makes PSP anthocyanins unique and also provides some advantages in $\mathrm{pH}$

39 and heat resistances, light sensitivity, and overall stability. From a nutritional viewpoint, acylated

40 anthocyanins have been reported to possess elevated antioxidant and anti-mutagenicity activity

41 (Suda et al., 2002). Biological activities of specific acyl groups are still under evaluation,

42 however, it is believed that additional free phenolic hydroxyl groups may raise bio-functionality

43 of anthocyanins. Among six common anthocyanidins peonidin and cyanidin are usually found in

44 PSP, but the most abundant anthocyanins in the reported PSPs were peonidin derivatives

45 (Yoshinaga et al. 1999). In addition, pelargonidin was a negligible anthocyanidin found in a few

46 varieties of PSP such as NC415 and Ayamurasaki (Giusti et al., 1999; Kim et al., 2012; Truong

47 et al., 2010). As an acylated anthocyanin source, PSP has shown excellent coloring properties in

48 numerous acidic to neutral foods that close to the synthetic FD\&C red \#40 (Suda et al., 2003). In

49 Japan, PSP puree is a popular natural colorant and functional ingredient in the bakery,

50 confectionery, juices, beverages, and dairy food industries (Dyrby et al., 2001; Giusti \& 
51 Wrolstad, 2003; Suda et al., 2003). In order to be used as an additive, PSP is usually transformed

52 to cooked puree, dried, and powdered; those processes lead to pigment degradation along

53 thermal treatments and oxygen exposure (Steed \& Truong, 2008). Therefore, thermal stability of

54 anthocyanin is recognized as a key property that affects overall quality.

P40 is a variety of anthocyanin-enriched PSP cultured at the John C. Pair

56 Horticulture Research Center, Wichita, KS, by selecting from a large number of parent-seedlings

57 provided by the International Potato Center in Lima, Peru. We previously reported P40

58 anthocyanins at 10-40 $\mu \mathrm{M}$ significantly inhibited the growth of the human colon cancer SW480

59 cells by arresting cell cycle phase at G1 (Lim et al., 2013). Mice fed 10-30\% of P40 showed less

60 susceptible to azoxymethane-induced colorectal aberrant crypt foci formation, demonstrating a

61 potential cancer prevention (Lim et al., 2013). The objective of this follow-up study is to

62 characterize anthocyanin contents and profile in P40. The thermal stability of them during

63 various cooking conditions is further evaluated.

\section{2. Materials and methods}

\section{2.1. Chemicals}

66 Acetonitrile, methanol, hexane, and formic acid were either HPLC grade or analytic

67 grade purchased from Thermal Fisher Scientific (Suwanee, GA). Water used in all preparation

68 and analysis was purified through Barnstead E-Pure Deionization System (Dubuque, IA) and

69 filtered using Millipore $0.45 \mu \mathrm{m}$ membrane (Bedford, MA). Standards of cyanidin-3, 5-

70 diglucoside was obtained from Sigma-Aldrich (St. Louis, MO).

71 2.2. Sample preparation and cooking condition

72 The PSP P40 is a variety of anthocyanin-rich sweet potato selected and cultured at the

73 John C. Pair Horticulture Research Center, Wichita, KS. Average weight 120-150 g of intact root 
74 tubers were skinned and cut to $6.4 \mathrm{~mm}$ thick wedges. Various cooking conditions based upon

75 conventional recipes include baking in conventional oven at $205^{\circ} \mathrm{C}$ for $50 \mathrm{~min}$, steaming in

76 Hamilton Beach rice cooker with steaming sleeve at $100{ }^{\circ} \mathrm{C}$ for $20 \mathrm{~min}$, pressure cooking in

77 Cuisinart pressure cooker at $121^{\circ} \mathrm{C}$ and 15 psi for $17 \mathrm{~min}$, microwave baking in conventional

$78850 \mathrm{~W}$ microwave at $100 \%$ power for $5 \mathrm{~min}$, and frying in conventional deep fryer at $177{ }^{\circ} \mathrm{C}$ for

$795 \mathrm{~min}$. The cooked tubers were peeled, freeze-dried, and powdered using a mortar and pestle. An

80 additional de-fatting procedure was performed on fried samples in case that the residual oil might

81 interfere with the later analyses.

82 2.3. Proximate Analysis

83 Lyophilized P40 powder was used for analyses of moisture, crude protein, lipid, and ash.

84 Briefly, moisture was removed by hot oven method at $130{ }^{\circ} \mathrm{C}$ for two hours; protein was

85 determined by a Leco FP-2000 protein analyzer (Leco Corp, St Joseph, MI, USA) using AOAC

86 method 992.15 with 6.25 as the converting factor (King-Brink \& Sebranek, 1993); crude lipid

87 and moisture was determined in CEM Smart Trac system (CEM Corporation, Matthews, NC,

88 USA) by AOAC method 2008.06 (Leffler et al., 2008); and ash content was determined using a

89 muffle furnace according to AOAC method 942.15 (Thiex, Novotny, \& Crawford, 2012).

90 2.4. Extraction and Quantification

91 Defatting: Powdered and fried PSP was extracted in hexane at solid to solvent ratio (1:6,

$92 \mathrm{w} / \mathrm{v})$ for one $\mathrm{hr}$, and centrifuged $\left(3000 \mathrm{~g}, 20 \mathrm{~min}, 4^{\circ} \mathrm{C}\right)$. The procedure was repeated three times

93 and the pellet was air dried overnight.

94 Anthocyanin Extraction: The extraction and subsequent analysis followed a method of

95 Kim et al., (2012), with minor modifications. Briefly, $1 \mathrm{~g}$ of the PSP powder containing internal

96 standard, cyanidin-3, 5-diglucoside, was extracted with $20 \mathrm{~mL} 5 \%$ formic acid water on an 
97 orbital shaker at $40{ }^{\circ} \mathrm{C}$ for $12 \mathrm{hrs}$ and centrifuged $\left(4000 \mathrm{~g}, 20 \mathrm{~min}, 4^{\circ} \mathrm{C}\right)$. The extraction was

98 repeated twice and the supernatants were pooled. A Waters Sep-Pak $\mathrm{C}_{18}$ solid phase extraction

99 cartridge (Milford, MA) was activated with $3 \mathrm{~mL}$ methanol and $3 \mathrm{~mL}$ water, and loaded with 2

$100 \mathrm{~mL}$ of supernatant. The colume was washed with $3 \mathrm{~mL}$ water and then anthocyanins were eluted

101 with $1 \mathrm{~mL}$ methanol, and the cartridge should be free of visible color after elution. The eluent

102 was evaporated in an Eppendorf Vacufuge (Hamburg, Germany) to dryness and re-constituted in

$1031 \mathrm{~mL}$ of $5 \%$ formic acid water.

$104 \quad$ 2.5. HPLC-MS/MS Analysis

105 HPLC coupled Electrospray Ionization tandem Mass Spectrometry (ESI/MS/MS) was

106 employed in this study as a proven powerful technique to carry out intact anthocyanin and

107 production-ion analysis. A Shimadzu HPLC system (Kyoto, Japan) was used for

108 chromatographic separation. This system employed a DGU-20A3 built in degasser, a LC-20AB

109 solvent delivery pump, a SIL-20ACHT auto-sampler, a CTO-20AC column holding oven, a

110 CBM-20A communicator module, and a SPD-M20A Photodiode Array Detectors. A Waters

111 (Milford, MA) $\mathrm{C}_{18}$ reversed phase column (250 mm length, $4.6 \mathrm{~mm}$ diameter) was used for

112 anthocyanin separation. Data was analyzed using LC Solution software (Kyoto, Japan). Elution

113 was performed with mobile phase A (5\% formic acid in de-ionized water) and mobile phase B

114 (5\% formic acid in acetonitrile/water 1:1 v:v); gradient expressed as mobile phase B volume was

$11520-40 \%$ for $30 \mathrm{~min}, 40-50 \%$ in following five min and held at 50\% for $10 \mathrm{~min}$ before returning

116 to $20 \%$. The flow rate was maintained as $1 \mathrm{~mL} / \mathrm{min}$ and column temperature was $25{ }^{\circ} \mathrm{C}$. Detector

117 performed a full spectrum scan between 190-800 nm where $525 \mathrm{~nm}$ was used for monitoring

118 anthocyanins. Cyanidin-3, 5-diglucoside was used as an internal standard for quantitation of

119 extraction recovery and anthocyanin contents. 
121 Mass spectrometric scan was performed in positive mode with a scanning interval 500-

$1221200 \mathrm{~m} / \mathrm{z}$. Nebulization was conducted at $350{ }^{\circ} \mathrm{C}$ aided by concurrent $\mathrm{N}_{2}$ flow at 10 psi; capillary

123 and cone voltages were set at $3.5 \mathrm{kV}$ and $40 \mathrm{~V}$; drying gas flow rate was $5 \mathrm{~L} / \mathrm{min}$. Mass of

124 precursor ions and reactions of fragments loss were evaluated. Data were analyzed using

125 BrukerHystar Post Processing software (Bruker, Bremen, Germany). Anthocyanin compounds

126 were identified by HPLC retention time, absorbance spectra pattern, and matching MS fragment

127 database according to previous publications (Giusti et al., 1999; Kim et al., 2012; Tian et al.,

128 2005a; Tian et al., 2005b; Truong et al., 2010).

129 2.7. Acid Hydrolysis

130 Acid hydrolysis procedure was adopted from Truong et al. (2010). A $50 \mu \mathrm{L}$ anthocyanin

131 extract was mixed with same volume of $6 \mathrm{~N} \mathrm{HCl}$ in a sealed HPLC vial. The mixture was heated

132 in boiling water bath for $2 \mathrm{hrs}$ after thorough vortexing. The sample was cooled in ice bath and

133 dried in an Eppendorf Vacufuge before re-constituted in $500 \mu \mathrm{L}$ of water acidified with $5 \%$

134 formic acid. Mass spectrometric scanning was performed at $100-800 \mathrm{~m} / \mathrm{z}$ range to identify

135 aglycone anthocyanidins.

136 2.8. Statistical Analysis

137 The anthocyanin change was analyzed by one-way ANOVA where cooking conditions

138 were main factors. Tukey's post-hoc test was used to assess the multiple differences of

139 individual anthocyanin at various cooking conditions. A probability of $\mathrm{P} \leq 0.05$ was considered

140 significant. Statistical procedures were by SAS 9.3 (SAS Institute; Cary, NC).

141 3. Results

142 3.1. Proximate analysis 
144 fat, and ash contents were determined as $8.4 \%, 0.7 \%$, and $4.3 \%$, respectively. Thermal

145 treatments resulted in leaching and drying effects and affected on moisture contents of fresh

146 sweet potato but did not alter the macronutrients after freeze drying (data not shown).

147 3.2. Chromatographic Separation

148 As shown in Figure 1, anthocyanin eluents were separated under the experimental 149 conditions. Twelve major peaks, possessed typical anthocyanin spectra of a maximum

150 absorbance at around $520 \mathrm{~nm}$, were separated in addition to internal standard, cyanidin-3,5-

151 diglucoside. Peaks 8, 9 and 10 were the major anthocyanins and they contributed to near half of

152 the total anthocyanin peak areas. Peak number, retention time, and \% of total peak areas were

153 summarized in Table 1.

154 3.3. Mass Spectrometric Identification

155 The $\mathrm{m} / \mathrm{z}$ ratio of each intact anthocyanin with daughter fragments were captured within

156 the scanning interval ranging. As shown in Figure 2A, the ions of peak 1, i.e., cyanidin 3-

157 sophoroside-5-glucoside (m/z 773), produced three fragments of $\mathrm{m} / \mathrm{z}$ 611, 449, and 287.

158 Transition $773>611$ and $773>449$ represented the loss of glucose $(\mathrm{m} / \mathrm{z} 162)$ and sophorose $(\mathrm{m} / \mathrm{z}$

159 324), respectively, while transition $773>287$ produced cyanidin ( $\mathrm{m} / \mathrm{z} 287)$ aglycone due to the

160 loss of both glucose and sophorose. Figure 2B showed another example for mono- and di-

161 acylated anthocyanin, i.e., cyanidin 3-p-hydroxybenzoyl sophoroside-5- glucoside (peak 2). The

162 ions of peak 2 produced transitions of 893 to 731,449 , and $287 \mathrm{~m} / \mathrm{z}$, where $893>449$ transition

163 indicated the loss of sophoroside and acylation. Identification of the remaining anthocyanins

164 (peaks 3-12) were carried out in a similar fashion. All the $\mathrm{m} / \mathrm{z}$ ratio of each intact anthocyanin

165 and its daughter fragments were summarized in Table 1. As indicated in Table 1, cyanidin $(\mathrm{m} / \mathrm{z}$ 
287) and peonidin (m/z 301) were two aglycone anthocyanidins detected. Glycosylations with

167 glucose (m/z 162) and sophorose (m/z 324) were found in all 12 anthocyanins. Eleven of them

168 except for cyanidin 3-sophoroside-5-glucoside (peak 1) were acylated at R1, R2, or R3 by caffeic

169 acid (m/z 180), ferulic acid (m/z 194), and/or p-hydroxybenzoic acid (m/z 138), respectively.

170 The chemical structures of skeleton anthocyanin and three acylated phenolic acids were shown in

171 Figure 3.

172 3.4. Acid Hydrolysis

173 Acid hydrolysis completely removed all the glycosylation attachments and reduced the

174 number of peaks from 9 to 3 (Figure 1). While the $3^{\text {rd }}$ peak was un-identified, peaks 2 and 3 were

175 simple cyanidin and peonidin, respectively, because they presented the $\mathrm{m} / \mathrm{z}$ ratio as identified by

176 MS at 287 and $301 \mathrm{~m} / \mathrm{z}$, respectively (data not shown). Both peak areas of cyanidin and peonidin

177 in the hydrolysate of raw P40 contributed to $90 \%$ of total peak area, but near $80 \%$ of total

178 anthocyanidins measured by acid hydrolysis were cyanidin derivatives.

179 3.5. Effect of Thermal Treatments

180 Total contents of anthocyanin in raw P40 and cooked P40 via various cooking conditions

181 were presented in Table 2. HPLC chromatograms of anthocyanins in baked and microwaved P40

182 in comparison with that in raw P40 were shown in Figure 1. The raw P40 possessed a content of

183 anthocyanins up to $14 \mathrm{mg} / \mathrm{g}$ dry matter. The top three major anthocyanins were peak 8 (cyanidin

184 3-caffeoyl-p-hydroxybenzoyl sophoroside-5-glucoside), peak 9 (peonidin 3-caffeoyl

185 sophoroside-5-glucoside), and peak 10 [cyanidin 3-(6"-caffeoyl-6"-feruloylsophoroside)-5-

186 glucoside], which account for half of the total anthocyanin content. Seven non-, mono-, or di-

187 acylated cyanidin species and five mono- or di-acylated peonidin species contribute for $67 \%$ and

$18833 \%$ of total anthocyanins, respectively. While baking did not affect total contents of 
anthocyanins significantly, steaming, high pressure cooking, microwaving, and frying reduced 8-

$19016 \%$ of total anthocyanin contents. Mono-acylated anthocyanins showed a higher resistance

191 against heat than di- and non-acylated. Among of which, cyanidin 3-p-

192 hydroxybenzoylsophoroside-5-glucoside exhibits the best thermal stability.

\section{Discussion}

The objectives of this study were focused on characterizing anthocyanin profile in P40

195 and evaluated their thermal stability. HPLC-MS/MS analysis was applied for anthocyanin extract

196 of P40 before and after various thermal treatments, and then anthocyanin contents were assessed.

197 A total of 12 anthocyanins were identified and quantitated by HPLC-MS/MS. Eleven of

198 them were acylated with caffeic, ferulic, and/or p-hydrobenzoic acids. When compared to the

199 anthocyanin content of PSPs reported by others (Table 3), total anthocyanin in P40 was the

200 highest (near $1.4 \%$ in dry weight). If compared with berry fruits or colored vegetables, P40

201 ( 3000 mg/kg fresh weight) ranked at upper-middle of the ladder, but was still higher to

202 strawberry (350 mg/kg) and red cabbage (250 mg/kg) (Clifford, 2000). It was noteworthy that

203 anthocyanins in P40 were distinguishable from berry anthocyanins because of the unique

204 acylation that was presented in P40 only (Gould, Davies \& Winefield, 2008; Neto, 2007).

205 Identification of anthocyanins was greatly relied on the matching fragment patterns to the

206 mass spectrum database of anthocyanins collected from the published articles. The $\mathrm{m} / \mathrm{z}$ of the

207 precursor ions were detected by $1^{\text {st }}$ MS after electrospray ionization, and the precursor ions were

208 further dissociated by argon collision for $2^{\text {nd }}$ MS detection. During the collision, the glycosidic

209 bonds joining anthocyanidins and saccharides were vulnerable and cleaved; therefore, each

210 anthocyanin was identified by matching residual fragmentation. Furthermore, tandem MS

211 exhibited a distinct advantage in distinguishing similar isomers. For example, peak 5 [cyanidin 
212 3-(6" -feruloyl sophoroside)-5-glucoside] and peak 9 (peonidin 3-caffeoyl sophoroside-5-

213 glucoside) possessed a same molecular weight (m/z 949), but their identities were revealed by

214 different subsequent fragmentations.

215 PSPs are usually classified into either cyanidin-predominated or peonidin-predominated

216 based upon the ratio of peonidin to cyanidin aglycones (pn/cy). Pn/cy is an important factor to

217 flesh color and maybe some difference in functionalities. Peonidin type (pn/cy>1) sweet potatoes

218 generally have a pink to red flesh color and sometimes they are referred as red-fleshed. As

219 content of cyanidins increase, the color of the flesh shifts to purple and dark purple. Structure-

220 wisely, cyanidin contains more hydroxyl groups than peonidin, leading to a stronger

221 antioxidative activity. Yoshimoto et al. (1999a) reported cyanidin type pigments were superior to

222 peonidin in antioxidant and anti-mutagenicity. However, almost all the PSPs containing a high

223 content of anthocyanins were cyanidin-predominated type (Table 3). P40, as confirmed by acid

224 hydrolysis, was a unique cyanidin-predominated type with exceptionally high anthocyanin 225 content.

226 As a subgroup of polyphenolic flavonoids, anthocyanins are expected to be degraded by

227 heat (Xu \& Chang, 2008a). There was a significant impact on anthocyanin contents after various

228 thermal treatments. While baking slightly reduced total contents of anthocyanins, steaming, high

229 pressure cooking, microwaving, and frying significantly reduce 8-16\% of total anthocyanin

230 contents. It appeared that each anthocyanin varied greatly in its thermal stability, and most likely

231 the acylation played a role. It appeared that mono-acylated anthocyanin with p-hydroxybenzoic

232 acid possessed the best resistance against heating, followed by ferulic acid and caffeic acid

233 acylation. Mono-acylated anthocyanins generally showed a higher resistance against heat than

234 di- and non-acylated. Among of which, cyanidin 3-p-hydroxybenzoylsophoroside-5-glucoside 
235 exhibits the best thermal stability. Therefore, mono-acylated and cyanidin-predominated

236 anthocyanins in P40 might be more stable in resistance against thermal treatments. Furthermore,

237 thermal treatments may release phytochemicals by destroying and softening bound from food

238 matrices (Xu \& Chang, 2009). In the microwave treatment, for example, cyanidin 3-p-

239 hydroxybenzoylsophoroside-5-glucoside (peak 2) increased from 121 to $462 \mathrm{mg} / 100 \mathrm{~g}$ and

240 peonidin 3-p-hydroxybenzoyl sophoroside-5-glucoside (peak 4) was elevated from 19 to 87

$241 \mathrm{mg} / 100 \mathrm{~g}$. It seemed that microwave treatment might release anthocyanins from physical

242 entrapment in other structures as suggested by Xu \& Chang (2008b).

\section{5. Conclusions}

244 Twelve individual anthocyanins were identified and quantified in the newly bred purple

245 sweet potato P40, where eleven were acylated and seven were cyanidin derivatives. Top three

246 main anthocyanins in P40 were cyanidin 3-caffeoyl-p-hydroxybenzoyl sophoroside-5-glucoside,

247 peonidin 3-caffeoyl sophoroside-5-glucoside, and cyanidin 3-(6" -caffeoyl-6"-

248 feruloylsophoroside)-5-glucoside, which account for half of the total anthocyanin contents. Over

$24980 \%$ of total anthocyanins measured by acid hydrolysis were cyanidin derivatives. To the best of

250 our knowledge, P40 seems the first cyanidin-predominated purple-fleshed sweet potato with

251 superior anthocyanin contents. While conventional baking did not reduce anthocyanin content

252 significantly, other thermal treatments facilitated anthocyanin degradation about 8-16\% of total

253 anthocyanin contents. Mono-acylated anthocyanins show a higher resistance against heat than di-

254 and non-acylated. Among of which, cyanidin 3-p-hydroxybenzoylsophoroside-5-glucoside

255 exhibits the best thermal stability. Therefore, mono-acylated and cyanidin-predominated

256 anthocyanins in P40 appeared stable in resistance against thermal treatments, which may be an 
257 advantage to the development of a functional sweet potato product for chronic disease

258 prevention.

\section{ACKNOWLEDGEMENTS}

260 This study was supported in part by a USDA Cooperative Project KS410-0214022 from

261 the Agricultural Experiment Station, Kansas State University (Contribution \#14-320-J from the 262 Kansas Agricultural Experiment Station). 


\section{References}

264 Clifford, M. N. (2000). Anthocyanins - nature, occurrence and dietary burden. Journal of the Science of Food and Agriculture, 80(7), 1063-1072.

Dyrby, M., Westergaard, N., \& Stapelfeldt, H. (2001). Light and heat sensitivity of red cabbage extract in soft drink model systems. Food Chemistry, 72(4), 431-437.

Giusti, M. M., Rodríguez-Saona, L. E., Griffin, D., \& Wrolstad, R. E. (1999). Electrospray and tandem mass spectroscopy as tools for anthocyanin characterization. Journal of Agricultural and Food Chemistry, 47(11), 4657-4664.

271 Giusti, M., \& Wrolstad, R. E. (2003). Acylated anthocyanins from edible sources and their applications in food systems. Biochemical Engineering Journal, 14(3), 217-225.

273 Gould, K., Davies, K. M., \& Winefield, C. (2008). Anthocyanins: Biosynthesis, Functions, and 274 Applications (2009 edition.). New York: Springer.

Kim, H. W., Kim, J. B., Cho, S. M., Chung, M. N., Lee, Y. M., Chu, S. M., Che, J. H., Kim S. N., Kim, S. Y., Cho, Y. S., Kim, J. H., Park, H. J., \& Lee, D. J. (2012). Anthocyanin changes in the Korean purple-fleshed sweet potato, Shinzami, as affected by steaming and baking. Food Chemistry, 130(4), 966-972.

King-Brink, M., \& Sebranek, J. G. (1993). Combustion method for determination of crude protein in meat and meat products: collaborative study. Journal of AOAC International, 76(4), 787-793.

282 Leffler, T. P., Moser, C. R., McManus, B. J., Urh, J. J., Keeton, J. T., \& Claflin, A. (2008).

283 Determination of moisture and fat in meats by microwave and nuclear magnetic resonance analysis: collaborative study. Journal of AOAC International, 91(4), 802-810. 
Lim, S., Xu, J., Kim, J., Chen, T.-Y., Su, X., Standard, J., Edward, C., Jason, G., Betty, H., Katz, B., Tomich, J., \& Wang, W. (2013). Role of anthocyanin-enriched purple-fleshed sweet potato P40 in colorectal cancer prevention. Molecular Nutrition \& Food Research,

Neto, C. C. (2007). Cranberry and blueberry: Evidence for protective effects against cancer and 57(11), 1908-1917.

\section{1}

292

293

294

295

296

297

298

299

300

301

302

303

304

305

306 vascular diseases. Molecular Nutrition \& Food Research, 51(6), 652-664.

Rodriguez-Saona, L. E., Giusti, M. M., \& Wrolstad, R. E. (1998). Anthocyanin pigment composition of red-fleshed potatoes. Journal of Food Science, 63(3), 458-465.

Steed, L. E., \& Truong, V.-D. (2008). Anthocyanin content, antioxidant activity, and selected physical properties of flowable purple-fleshed sweetpotato purees. Journal of Food Science, 73(5), S215-221.

Suda, I., Oki, T., Masuda, M., Kobayashi, M., Nishiba, Y., \& Furuta, S. (2003). Physiological functionality of purple-fleshed sweet potatoes containing anthocyanins and their utilization in foods. Japan Agricultural Research Quarterly, 37(3), 167-173.

Suda, I., Oki, T., Masuda, M., Nishiba, Y., Furuta, S., Matsugano, K., Sugita, K., \& Terahara, N. (2002). Direct absorption of acylated anthocyanin in purple-fleshed sweet potato into rats. Journal of Agricultural and Food Chemistry, 50(6), 1672-1676.

Thiex, N., Novotny, L., \& Crawford, A. (2012). Determination of ash in animal feed: AOAC official method 942.05 revisited. Journal of AOAC International, 95(5), 1392-1397.

Tian, Q., Giusti, M. M., Stoner, G. D., \& Schwartz, S. J. (2005a). Screening for anthocyanins using high-performance liquid chromatography coupled to electrospray ionization tandem mass spectrometry with precursor-ion analysis, product-ion analysis, common-neutral- 
loss analysis, and selected reaction monitoring. Journal of Chromatography. A, 1091(12), 72-82.

Tian, Q., Konczak, I., \& Schwartz, S. J. (2005b). Probing anthocyanin profiles in purple sweet

Truong, V.-D., Deighton, N., Thompson, R. T., McFeeters, R. F., Dean, L. O., Pecota, K. V., \& potato cell line (Ipomoea batatas L. Cv. Ayamurasaki) by high-performance liquid chromatography and electrospray ionization tandem mass spectrometry. Journal of Agricultural and Food Chemistry, 53(16), 6503-6509.

Yencho, G. C. (2010). Characterization of anthocyanins and anthocyanidins in purplefleshed sweetpotatoes by HPLC-DAD/ESI-MS/MS. Journal of Agricultural and Food Chemistry, 58(1), 404-410.

Wu, X., Sun, C., Yang, L., Zeng, G., Liu, Z., \& Li, Y. (2008). $\beta$-carotene content in sweet potato varieties from China and the effect of preparation on $\beta$-carotene retention in the Yanshu No. 5. Innovative Food Science \& Emerging Technologies, 9(4), 581-586.

Xu, B., \& Chang, S. K. C. (2008a). Characterization of phenolic substances and antioxidant properties of food soybeans grown in the North Dakota-Minnesota region. Journal of Agricultural and Food Chemistry, 56(19), 9102-9113.

Xu, B., \& Chang, S. K. C. (2008b). Total phenolics, phenolic acids, isoflavones, and anthocyanins and antioxidant properties of yellow and black soybeans as affected by thermal processing. Journal of Agricultural and Food Chemistry, 56(16), 7165-7175.

Xu, B., \& Chang, S. K. C. (2009). Phytochemical profiles and health-promoting effects of coolseason food legumes as influenced by thermal processing. Journal of Agricultural and Food Chemistry, 57(22), 10718-10731. 
329 Yoshimoto, M., Okuno, S., Yamaguchi, M., \& Yamakawa, O. (2001). Antimutagenicity of deacylated anthocyanins in purple-fleshed sweetpotato. Bioscience, Biotechnology, and

332 Yoshinaga, M., Yamakawa, O., \& Nakatani, M. (1999). Genotypic Diversity of Anthocyanin Content and Composition in Purple-Fleshed Sweet potato (Ipomoea batatas (L.) Lam). Breeding Science, 49(1), 43-47.

335 Zhu, F., Cai, Y.-Z., Yang, X., Ke, J., \& Corke, H. (2010). Anthocyanins, hydroxycinnamic acid derivatives, and antioxidant activity in roots of different Chinese purple-fleshed sweetpotato genotypes. Journal of Agricultural and Food Chemistry, 58(13), 7588-7596. 
339 Figure 1. HPLC chromatograms of anthocyanins in raw, baked, microwaved, and acid 340 hydrolyzed P40.

341 Figure 2. Mass spectra of cyanidin 3-sophoroside-5-glucoside and cyanidin 3-p-hydroxybenzoyl

342 sophoroside-5- glucoside (peak 1 and 2 in Figure 1, respectively). A: a, b and a+b: bond cleavage

343 fragments without glucoside, sophoroside, and glucoside + sophoroside, respectively; B: a, b,

344 and $\mathrm{a}+\mathrm{b}$ : bond cleavage fragments without glucoside, $\mathrm{p}$-hydroxybenzoyl sophoroside, and

345 glucoside + p-hydroxybenzoyl sophoroside, respectively.

346 Figure 3. Skeleton structures of cyanidin $\left(\mathrm{R}_{1}=\mathrm{H}\right)$ or peonidin $\left(\mathrm{R}_{1}=\mathrm{CH}_{3}\right)$ 3-sophoroside-5-

347 glucoside (a), caffeic acid (b), ferulic acid (c), and p-hydrobenzoic acid (d). 

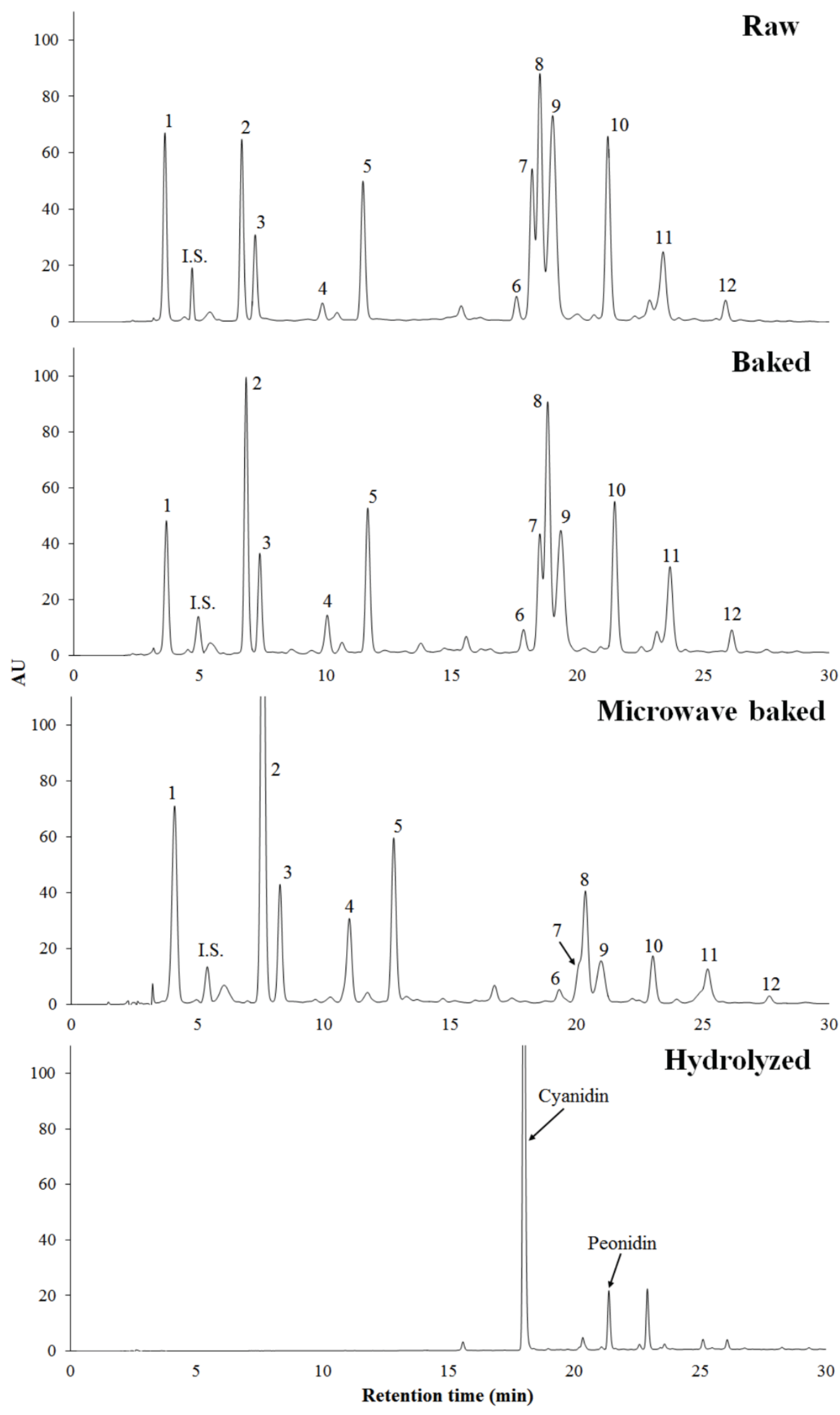


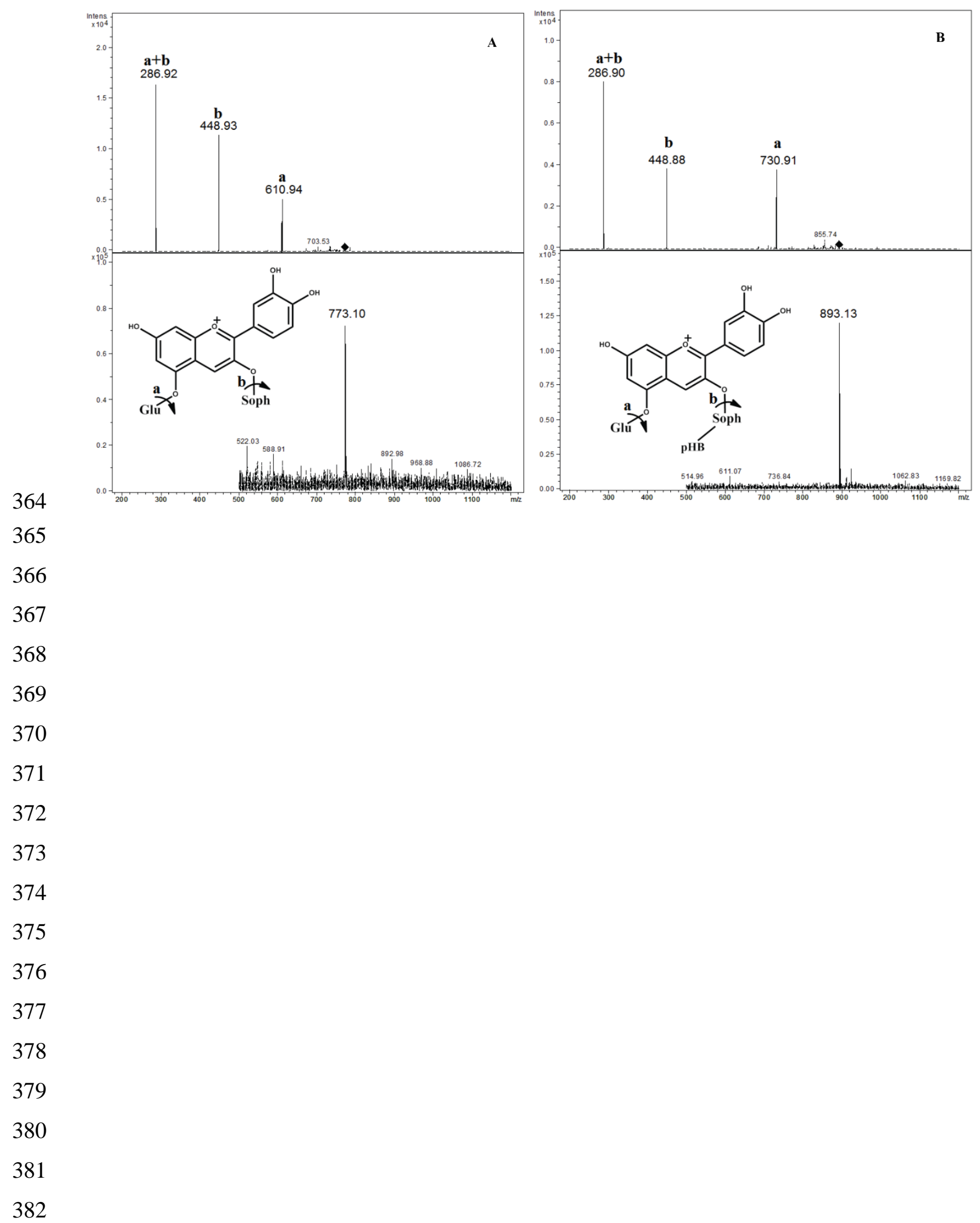




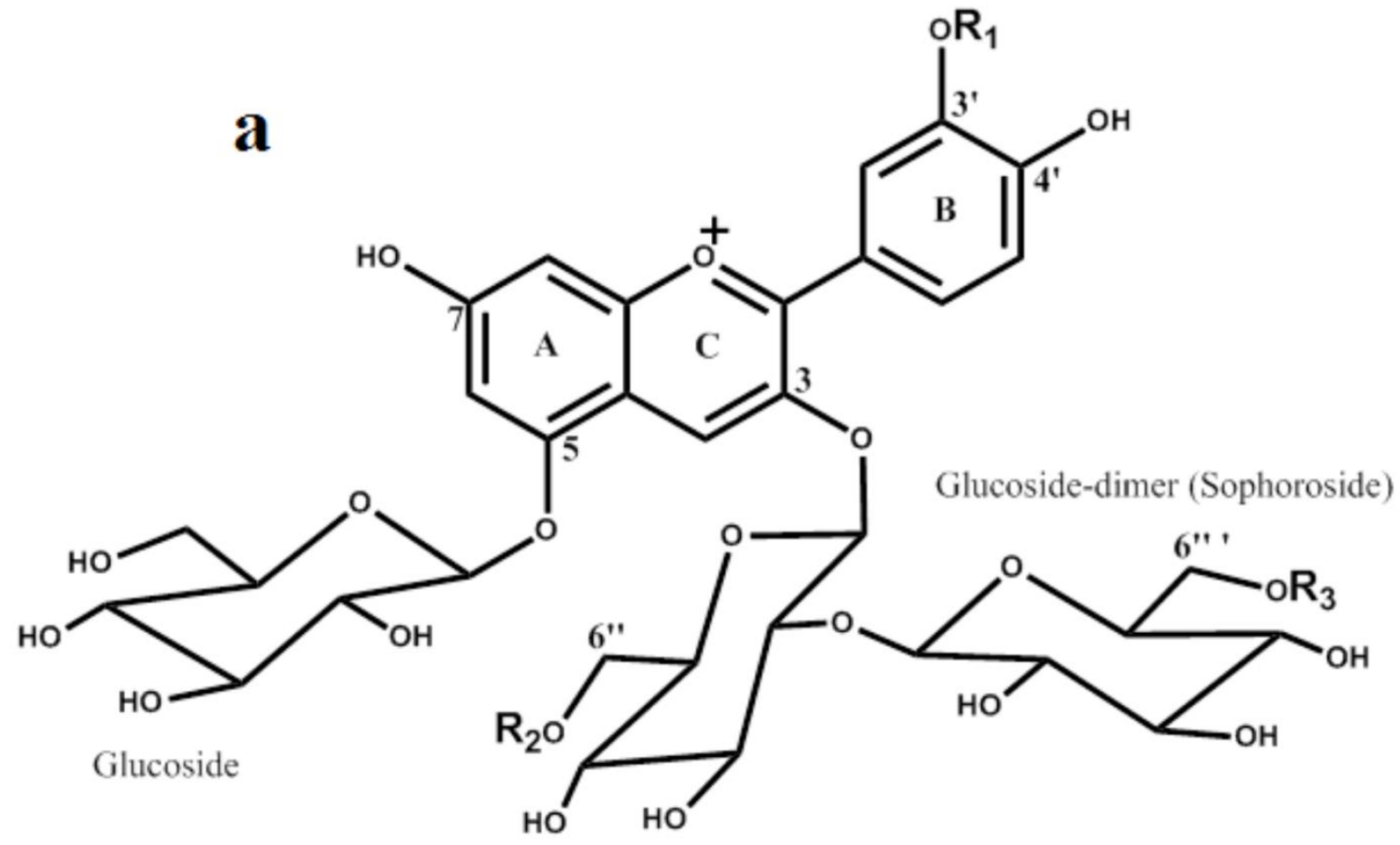<smiles>O=C(O)/C=C/c1ccc(O)c(O)c1</smiles> 